\title{
SIMULAÇÃO DO PROCESSO DE PRODUÇÃO DE INSERTO COM O SOFTWARE ARENA
}

\author{
Emmanuel Belga Rodrigues <emmanuel.rodrigues@alunos.unis.edu.br> \\ Maria Eduarda Belga Rodrigues<maria.belga@alunos.unis.edu.br> \\ Matheus Hernesto Mendes Alves <matheus.henesto@alunos.unis.edu.br> \\ Murilo Ramos Chiconelli <murilo.chiconelli@alunos.unis.edu.br> \\ Tiago Bittencourt Nazare<tiago@unis.edu.br>
}

\begin{abstract}
Resumo: No presente artigo foi utilizado o software arena para realizar uma simulação do processo de produção de mancais em uma fábrica de usinagem, localizada na cidade de Cataguases, interior de Minas Gerais, cujo o objetivo foi identificar possíveis melhorias e eliminar os gargalos encontrados. Deste modo, foram coletados os tempos médios de 3 operações antes do processo de montagem, adquiridos de um lote de 25 insertos. Assim, foi observado por meio da simulação realizada, que o tempo de operação sofreu uma redução de $29,14 \%$ utilizando o método de melhoria proposto.
\end{abstract}

\begin{abstract}
In this article, the arena software was used to simulate the bearing production process in a machining factory, located in the city of Cataguases, in the interior of Minas Gerais, whose objective was to identify possible improvements and eliminate bottlenecks found. In this way, the average times of 3 operations were collected before the assembly process, acquired from a batch of 25 inserts. Thus, it was observed through the simulation that the operating time was reduced by $29.14 \%$ using the proposed improvement method.
\end{abstract}


1. Introdução.

Diante o atual mercado globalizado e os constantes avanços tecnológicos, manter-se competitivo fez-se uma tarefa imprescindível para as atuais organizações conseguirem atender as demandas dos consumidores crescentemente exigentes. Para isso, é necessário que o processo torne-se eficiente e efetivo. Melhoria da qualidade, redução de custos, inovação e decisões ágeis são aspectos fundamentais para as atuais empresas.

O aprimoramento do processo é uma das medidas que as organizações podem tomar para obterem resultados competitivos e se sobressaíram sobre seus concorrentes. Contudo, é preciso ter o conhecimento de como as implementações adotadas irão afetar a empresa e assegurar que o investimento não cause um prejuízo.

O investimento em tecnologias, bem como em aprimorações e alterações de processos ou produtos, são consequentemente arriscados, já que os resultados podem não atender as expectativas pré-estabelecidas. Desta forma, tem-se o processo de modelagem, cujo seu objetivo é diminuir as incertezas e auxiliar na tomada de decisão para otimizar os processos. Harrel,et al (2002, p.2) define como "um processo de experimentação com um modelo de um sistema real para determinar como o sistema responderá a mudanças em sua estrutura, ambiente ou condições de contorno".

Com o intuito de identificar possíveis melhorias ou problemas relacionados a gargalos ou de layout, que resultam em uma produtividade ineficaz, este artigo buscou desenvolver o método de modelagem e simulação do processo produtivo.

Portanto, foi utilizado o software Arena desenvolvido por Rockwell Automation, com o objetivo de simular e modelar o processo de produção de incertos, peça sobressalente de mancais, em uma fábrica de usinagem localizada na cidade de Cataguases no interior de MInas Gerais.

2. Revisão de Literatura.

\subsection{Pesquisa Operacional}


Com o avanço de pesquisas no contexto da Segunda Guerra Mundial no final do século $X X$, a pesquisa operacional (PO) surgiu com o objetivo de apoiar a defesa aérea Britânica de forma a otimizar a utilização de radares (PASSOS, 2008).

Posteriormente em 1947, George Dantzig desenvolveu o método Simplex que permitiu a PO ser utilizada no cenário econômico em organizações como apoio na tomada de decisão (PASSOS, 2008).

A PO é a disciplina aplicada que utiliza modelos matemáticos para otimizar o processo das empresas, no qual são resolvidos com a assistência de ferramentas computacionais, uma vez que envolve um grande número de cálculos repetitivos. As disciplinas que complementam a análise da PO, conforme DÁVALOS, 2002, são matemática, informática, economia e estatística.

De acordo com DÁVALOS, 2002, alguns dos experimentos de PO são modelagem, solução e análise de problemas decisórios, logística de transportes, otimização de variáveis entre outras. Segundo Passos (2008), o objetivo da utilização é fornecer soluções efetivas de determinado problema para auxílio da tomada de decisão dos profissionais responsáveis.

\subsection{Processos}

De acordo com a FNQ (Fundação Nacional da Qualidade), podemos definir processo como um grupo de atividades que se inter-relacionam para transformar os insumos (entradas) em produtos finais (saídas). Utilizando uma abordagem técnica, são atividades preestabelecidas que possuem uma sequência já determinada para execução.

Mais do que implementar sistemas de trabalho com descrições e medições de cada atividade, a Gestão POR Processos necessita de visão sistêmica. Sem ela, é impossível perceber como o todo significa muito mais do que uma simples soma das partes. Diferentemente da tradicional Gestão DE Processos, que prevê os detalhes em cada sistema em operação, uma Gestão POR Processos pode ser descrita como um trabalho dinâmico e em rede (MOTA, p. 8).

Para a obtenção do pleno domínio dos recursos empregados pela empresa, bem como a previsibilidade dos seus resultados, a melhoria do seu desempenho e a implementação sistemática de inovações e melhorias, é orientado que a organização compreenda o gerenciamento por meio de processos (MOTA, p. 6). 


\subsection{Modelagem}

Para BORSHCHEV; FILIPPOV, 2004, modelagem nada mais é que uma maneira de resolver problemas do mundo real.

Siebers, et al. (2007) afirma que a modelagem é uma cópia limitada do verdadeiro mundo, e deve ser construída por meio de componentes significantes para que se construa um modelo eficiente.

Modelos consistem em representar determinada situação do mundo real, no qual por meio destas representações é possível o planejamento e previsões de atividades (CAIXETA-FILHO, 2004).

Já Prado (2009), diz que o modelo representa um sistema, sendo este podendo ser de base simbólica, quando tem como significado uma semelhança conceitual com o sistema em estudo, pode ser de base matemática, quando pode-se analisar por meio de equações matemáticas, ou diagramática, onde o sistema possui uma representação por diagramas. Ainda define que "o objetivo da modelagem de sistemas é, conhecer o cenário, as características e as necessidades de todos os envolvidos, obter o melhor dimensionamento." (PRADO 2009, p. 16)

De acordo com Harrel et al (2002, p. 1) "o modelo põe os componentes do sistema de uma forma tal que, se é capaz de compreender a realidade baseados em fenômenos conhecidos e permite realizar experimentos que ajudam a prever o comportamento do sistema real". Assim, o pode-se entender o modelo como uma maneira de simular eventos de uma situação real por meio de representações dos fenômenos conhecidos, permitindo fazer experimentos no sistema com a finalidade de encontrar resultados que auxiliem na tomada de decisão.

\subsection{Tomada de Decisão}

Para manter-se competitivo no mercado atual é Imprescindível que os responsáveis tomem decisões eficazes, Laudon (2014) afirma que decisões que baseiam-se em palpites e previsões sem fundamento pode gerar um sistema improdutivo ou em uma produção excessiva de bens e serviços, resultando em custos altos e clientes insatisfeitos.

Desta forma, com o intuito de evitar uma produção ineficiente, a tomada de decisão deve ser auxiliada por ferramentas como a PO e suas disciplinas. Para Guimarães (2004), a tomada de decisão deve ser resultado de um processo 
sistematizado do estudo do problema, mediante o levantamento dos dados, produção de informação e posterior geração de conhecimento, para em seguida viabilizar e implementar a decisão de forma a analisar os resultados obtidos.

\subsection{Programação Linear}

De acordo com Passos (2008), programação linear trata-se de uma técnica de otimização para sistemas de equações e inequações lineares, de forma a maximizar ou minimizar uma função sujeita a restrições, assim dizendo, por meio de modelos matemáticos, o intuito da programação linear é determinar valores de variáveis que buscam maximizar ou minimizar uma função, onde estes modelos possuem a finalidade de descrever o problema e auxiliar na tomada de decisão.

Hiller e Lieberman (2013), dizem que um modelo matemático de um problema de negócios é o sistema de equações que define sua essência. Para Silva (2011), diante um problema gerencial, existem duas maneiras para encontrar a solução ótima, usar a intuição gerencial ou fazer uso de modelos matemáticos.

Com os modelos se tornando mais complexos, a utilização de ferramentas computacionais para simulação tornou-se indispensável. A simulação é uma técnica de solução de um problema pela análise de um modelo, que descreve o comportamento do sistema usando um computador digital (PRADO, 2003).

A simulação computacional permite aos analistas tomar decisões importantes a respeito de novos projetos baseados na visualização de algo que ainda não foi testado, no gerenciamento de processos sua contribuição promove melhorias na resolução de problemas que ainda irão surgir. Para que a simulação contribua de forma positiva todas as variáveis que influenciam no modelo devem ser consideradas, fornecendo assim uma visão fiel do processo (AGUILAR et al, 2009). Paragon (2005) corrobora dizendo que a simulação é capaz de apresentar valores finais sem que seja feito qualquer alteração no quadro atual da organização, notificando tomadas de decisões e melhoramento de desempenho.

De acordo com Neto et al. (2014) a simulação utiliza métodos computacionais que possibilita simular um comportamento real e fazer alterações - ou manipular - tal sistema a fim de avaliar a execução.

A principal vantagem de utilizar a simulação é o fato de que a implementação de sistemas ou processos novos, bem como alterações dos mesmos, gera-se um custo, podendo causar prejuízos se não for bem planejado ou se as decisões 
tomadas pelos responsáveis não forem bem estruturadas. Prado (2009, p. 100) afirma que uma

"situação em que alterar o sistema existente, sem ter uma certeza de que a alteração vai dar certo, pode significar um alto risco de prejuízo". Gavira (2003, p. 57) diz que "utiliza-se de um modelo para estudar o sistema real, sem a necessidade de gastos com pessoal e equipamentos, com riscos de falhas reduzidos".

\subsection{Software ARENA}

O software Arena foi criado através da união de dois outros programas denominados SIMAN e CINEMA. De acordo com Prado (2004), o SIMAN é uma linguagem de simulação para computadores pessoais, sendo o primeiro programa voltado para essa área no ano de 1983. O CINEMA foi criado em 1984, sendo o primeiro programa para animação de simulação em computadores. Com a união e aprimoramento destes dois softwares foi originado o programa Arena em 1993.

O ARENA, de acordo com o Paragon Decision Science, oferece a possibilidade da criação de templates, sendo uma coleção de objetos/ferramentas de modelagem que possibilita ao utilizador retratar o comportamento do processo a ser analisado, através de respostas às perguntas pré-elaboradas, sem programação, de maneira visual e interativa. Através da utilização de templates, o software pode se transformar em um simulador específico.

Paragon (2005) afirma que o Arena é a composições de vários padrões que compõem a formação da modelagem desejada, são conhecidos de templates, que nada mais é do que um grupo de elementos que ajudam a ilustrar por meio de fluxograma o cenário atual da área semelhante.

\subsection{Layout}

Originalmente, o termo "layout" refere-se ao arranjo físico dos equipamentos. Hoje, o termo generalizou-se, e o seu objetivo principal representa o melhor uso do espaço, que resulta em um processo mais efetivo, por pequenas distâncias no menor tempo (Toledo, 2004, p. 8).

Conforme Nunes et al. (2012), o layout de uma empresa deve condizer com sua estratégia, pois existem arranjos físicos que são direcionados para melhorar a 
flexibilidade, outros viabilizam a customização de produtos, e outros possuem a função de melhorar a eficiência da empresa, melhorando os fluxos dos recursos ao longo do processo de produção.

A configuração de um layout determina a forma de organizar as máquinas e departamentos da empresa de modo a minimizar o tempo de ciclo do produto dentro da fábrica, gerando a maximização do volume de negócios e da capacidade produtiva a partir da redução de distâncias percorridas (ROSA et al., 2014).

\section{Materiais e métodos}

A pesquisa foi realizada em uma empresa de usinagem de mancais, localizada na cidade de Cataguases, interior de Minas Gerais, para realização da pesquisa foram levantados os tempos médios de 3 operações antes do processo de montagem, os tempos coletados levam em consideração um lote de 25 insertos, composto por duas metades que foram usinadas em um centro de usinagem que possui um pallet duplo, permitindo preparar a próxima peça durante a operação da peça atual, portanto os tempos são referentes a operações de furação inferior, furação superior e rasgo superior deste mesmo lote e um tempo de preparação da máquina de 45 minutos. Para coleta de tempo foi utilizado um aplicativo de cronômetro em um celular modelo moto g7. Os dados coletados foram colocados em uma tabela e depois utilizados no simulador de processos Arena.

\subsection{Dados coletados}

Todos os dados de tempo coletados pelo pesquisador levam em consideração os tempos de parada da máquina durante a operação de algumas peças, por isso, pode haver variações de tempo para mais ou para menos, contudo essa variação não será prejudicial, uma vez que essa variação é dada em segundos e não extrapolam o tempo médio obtido. 


\begin{tabular}{|c|c|c|c|c|c|}
\hline \multicolumn{2}{|c|}{ Furação da Metade Inferior } & \multicolumn{2}{|c|}{ Furação da Metade Superior } & \multicolumn{2}{|r|}{ Rasgo } \\
\hline Peça & Tempo em minutos & Peça & Tempo em minutos & Peça & Tempo em minutos \\
\hline 1 & $00: 29: 15$ & 1 & $00: 25: 12$ & 1 & $00: 18: 11$ \\
\hline 2 & 00:29:30 & 2 & $00: 24: 46$ & 2 & 00:18:06 \\
\hline 3 & $00: 28: 35$ & 3 & $00: 24: 30$ & 3 & $00: 17: 26$ \\
\hline 4 & $00: 29: 15$ & 4 & $00: 25: 20$ & 4 & 00:18:19 \\
\hline 5 & 00:28:32 & 5 & $00: 23: 46$ & 5 & $00: 17: 33$ \\
\hline 6 & $00: 29: 11$ & 6 & $00: 25: 03$ & 6 & $00: 17: 47$ \\
\hline 7 & $00: 30: 06$ & 7 & $00: 23: 48$ & 7 & $00: 16: 58$ \\
\hline 8 & $00: 30: 13$ & 8 & $00: 24: 33$ & 8 & $00: 17: 20$ \\
\hline 9 & 00:29:09 & 9 & 00:25:30 & 9 & $00: 17: 39$ \\
\hline 10 & 00:30:01 & 10 & $00: 24: 48$ & 10 & $00: 17: 55$ \\
\hline 11 & $00: 30: 25$ & 11 & 00:23:59 & 11 & $00: 18: 30$ \\
\hline 12 & $00: 28: 56$ & 12 & $00: 24: 36$ & 12 & $00: 18: 26$ \\
\hline 13 & $00: 28: 47$ & 13 & $00: 24: 49$ & 13 & 00:18:01 \\
\hline 14 & $00: 30: 26$ & 14 & $00: 25: 13$ & 14 & 00:17:39 \\
\hline 15 & $00: 29: 26$ & 15 & $00: 23: 49$ & 15 & $00: 17: 36$ \\
\hline 16 & $00: 28: 50$ & 16 & $00: 24: 46$ & 16 & $00: 18: 30$ \\
\hline 17 & 00:28:59 & 17 & 00:25:04 & 17 & $00: 18: 12$ \\
\hline 18 & 00:29:39 & 18 & $00: 23: 38$ & 18 & $00: 17: 48$ \\
\hline 19 & $00: 29: 20$ & 19 & $00: 25: 15$ & 19 & $00: 17: 38$ \\
\hline 20 & $00: 30: 28$ & 20 & $00: 24: 48$ & 20 & $00: 18: 20$ \\
\hline 21 & $00: 28: 58$ & 21 & $00: 25: 30$ & 21 & $00: 17: 45$ \\
\hline 22 & $00: 29: 37$ & 22 & $00: 23: 48$ & 22 & $00: 18: 06$ \\
\hline 23 & $00: 28: 47$ & 23 & $00: 25: 01$ & 23 & $00: 17: 26$ \\
\hline 24 & $00: 28: 53$ & 24 & $00: 24: 36$ & 24 & $00: 16: 56$ \\
\hline 25 & $00: 29: 36$ & 25 & $00: 24: 22$ & 25 & $00: 17: 31$ \\
\hline
\end{tabular}

Tabela 1 - Tempos obtidos nas operações

Com os dados das operações foi possível realizar a simulação do layout de operação no software arena com o tempo médio de cada operação. 
3.2. Resultados e discussões

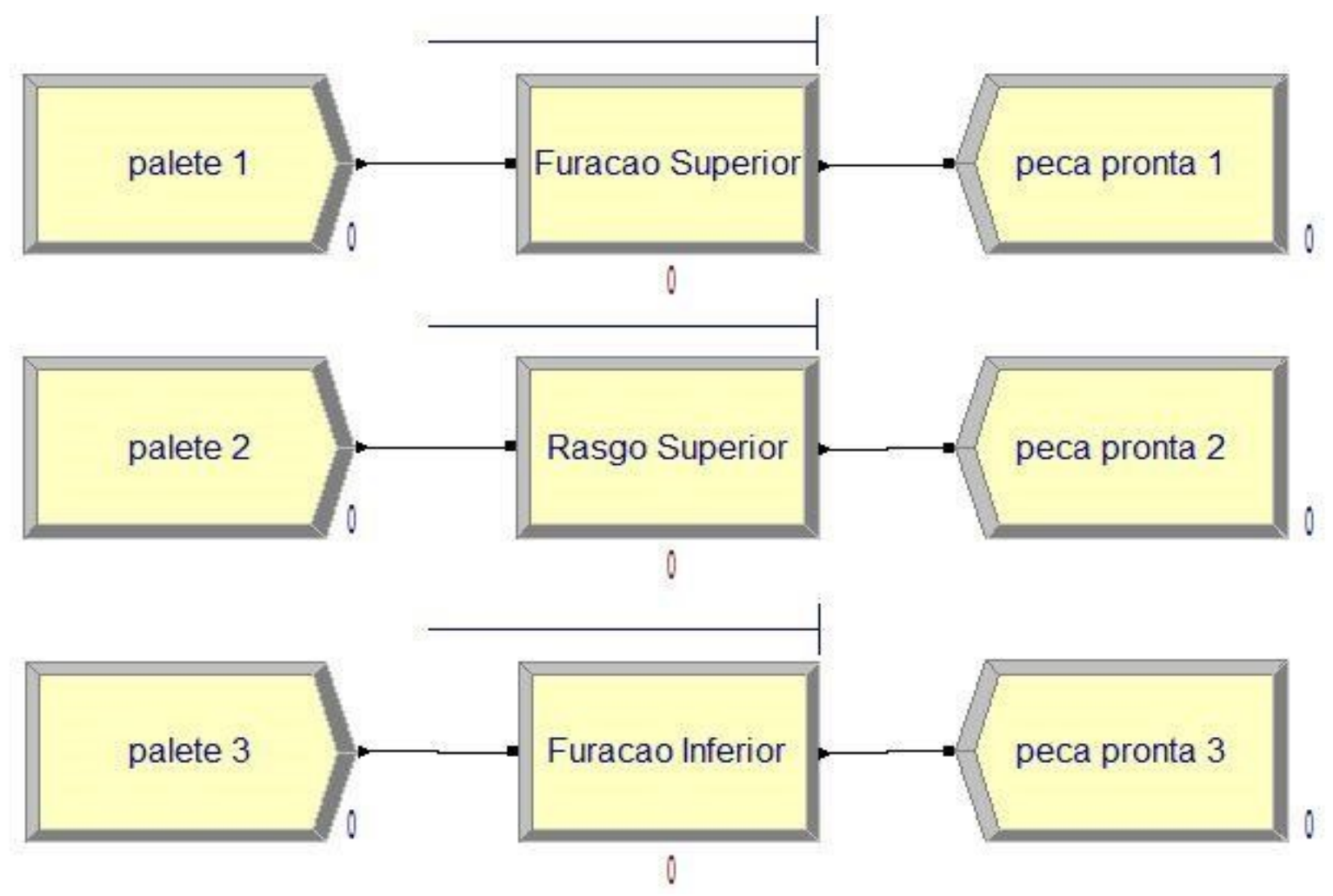

Figura 1 - Fluxograma de Operações

O modelo apresentado na figura 1 permitiu verificar o funcionamento de cada etapa do processo. Vale ressaltar que a simulação representa a produção de um lote de 25 peças e demonstra como a operação é feita em cascata, ou seja, cada operação depende da anterior acabar para poder ser iniciada, isso cria gargalos na linha de produção.

Os tempos coletados são apresentados na figura 2.

\begin{tabular}{lcccr} 
Total Time & Avergge & Half Width & $\begin{array}{r}\text { Minimum } \\
\text { Value }\end{array}$ & $\begin{array}{r}\text { Maximum } \\
\text { Value }\end{array}$ \\
\hline metade inferior & 364.70 & (Insufficient) & 28.8477 & 699.52 \\
Metade Superior & 300.32 & (Insufficient) & 23.7650 & 576.97 \\
Rasgo Superior & 210.50 & (Insufficient) & 16.6882 & 404.04
\end{tabular}

Figura 2 - Tempos de operação 
Durante o processo,a metade inferior do inserto passa apenas pelo processo de furação, já a metade superior por furação e rasgo. essa etapa do processo apresentou um tempo de operação total de 981,01 minutos.

Tendo em vista uma diminuição de tempo dessa etapa e por consequência uma duração menor da produção total, foi proposto uma nova simulação com duas máquinas.

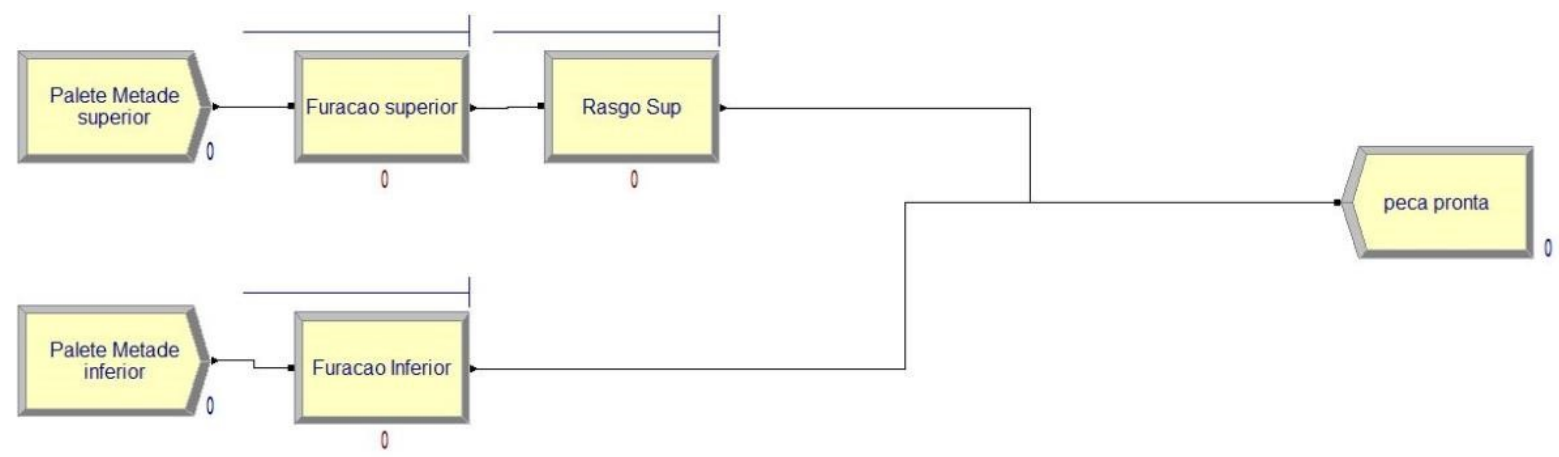

Figura 3 - Fluxograma de operações proposto

Com a identificação do gargalo ocasionado na metade superior, foi proposto um novo layout com a utilização de duas máquinas, a partir de agora a metade superior será furada em simultâneo com o rasgo (à medida que uma peça Superior fica pronta, ela é liberada para o rasgo) isso diminui o tempo e o gargalo criado nessa etapa, a metade inferior é furada depois que a metade superior e completada.

\begin{tabular}{lrrrr} 
Total Time & Averse & Half Width & $\begin{array}{r}\text { Minimum } \\
\text { Value }\end{array}$ & $\begin{array}{r}\text { Maximum } \\
\text { Value }\end{array}$ \\
\hline metade Inferior & 363.07 & (Insufficient) & 28.3418 & 695.17 \\
Metade superior & 313.29 & (Insufficient) & 40.7957 & 586.21
\end{tabular}

Figura 4 - Tempo de operação com a melhoria

É visto que na metade superior o tempo de operação é maior, uma vez que esta metade passa por duas operações diferentes, com o layout proposto é possível ver uma redução no tempo de operação da metade superior de $29,14 \%$ e consequentemente uma redução no tempo total de produção do inserto.

\section{Conclusão}

Após Simular os processos de produção, conclui-se que o arena é uma excelente ferramenta de gestão de produção, uma vez que, através dele foi possível verificar que o tempo total de operação era de 981,01 minutos na metade superior e a partir disso foi possível identificar este gargalo e propor as melhorias necessárias. 
Foi sugerido uma simulação com um novo layout que atingiu uma redução de $29,14 \%$ finalizando o processo com 586.21 minutos.

Vale ressaltar a importância de se observar os processos de produção , uma vez mapeados e compreendidos, será possível elaborar estratégias de melhorias.

Com a proposta de redução de tempo, os resultados obtidos na simulação foram suficientes para apresentar uma melhoria satisfatória na produção, porém vale salientar que uma versão aprimorada do software pode apresentar mais alternativas e soluções para a melhoria do processo.

\section{Referências Bibliográficas}

PASSOS, Eduardo José Pedreira Franco dos. Programação linear como instrumento da pesquisa operacional. São Paulo: Atlas, 2008.

DÁVALOS, R. V. Uma abordagem do ensino de pesquisa operacional baseada no uso de recursos computacionais. In: ENCONTRO NACIONAL DE ENGENHARIA DE PRODUÇÃO, 22., 2002, Curitiba. Anais eletrônicos...Curitiba: Enegep, 2002. 8 p.

BORSHCHEV, A.; FILIPPOV, A. From system dynamics and discrete event to practical agent based modeling: Reasons, techniques, tools. In:International Conference Of The System Dynamics Society. Jul 25-29, Oxford, England: Keble College, 2004.

SIEBERS, P.; AICKELIN, U; CELIA, H; CLEGG, C.W. Understanding retail productivity by simulating management practices.In: EUROSIM, Slovenia, 2007.

CAIXETA-FILHO, J.V. Pesquisa operacional:técnicas de otimização aplicadas a sistemas agroindustriais. 2. ed. São Paulo: Atlas, 2004.

PRADO, D.S.Teoria das filas e da simulação. 4. ed. Belo Horizonte: INDG, 2009 . Usando o Arena em simulação. 3.ed. Belo Horizonte: INDG, 2003. Usando o Arena em simulação. Belo Horizonte: INDG, 2004.

HARREL, C.R.; MOTT,J.R. A.; BATEMAN, R.E.;BOWDEN, R.G.; GOGG T.J. Simulação:otimizando os sistemas. 2.ed. São Paulo: IMAM, 2002.

LAUDON, Kenneth C.; LAUDON, Jane P. Sistemas de informações gerenciais. São Paulo: Pearson, $2014,11^{\mathrm{a} e d}$.

GUIMARÃES, Eliane Marina Palhares; ÉVORA, Yolanda Dora Martinez. Sistema de informação: instrumento para tomada de decisão no exercício da gerência.Scielo,Brasilia, v. 33, n. 1, p.72-80, abr. 2004. 
PASSOS, Eduardo José Pedreira Franco dos. Programação linear como instrumento da pesquisa operacional. São Paulo: Atlas, 2008.

HILLIER, Frederick S.; LIEBERMAN, Gerald J..Introdução à Pesquisa Operacional.9. ed. Porto Alegre: Amgh Editora Ltda, 2013. 1028 p.

SILVA, B. W. Pesquisa operacional: visão geral. In: BWS CONSULTORIA, 25 abr. 2011.

AGUILAR, S. M.S.; GUIMARÃES, I. F. G.; SCHUCHTER, D. C.; MENDES, L. G. Avaliação dos benefícios da aplicação da simulação, através do software arena

10.0, em uma empresa de transporte ferroviário. In: ENCONTRO NACIONAL DE ENGENHARIA DE PRODUÇÃO, 29.,2009, Salvador. Anais eletrônicos...Salvador: Enegep, 2009. 13p.

PARAGON. Introdução à Simulação. In: ENCONTRO NACIONAL DE ENGENHARIA DE PRODUÇÃO. Porto Alegre, 2005. Anais... Porto Alegre: ENGENEP, 2005.

NETO, H. G. et al. Modelagem e simulação computacional em uma empresa de confecção de roupas: um estudo sobre o desempenho de um processo produtivo. XXXIV ENEGEP, 2014.

GAVIRA, Murial de Oliveira. Simulação computacional como uma ferramenta de aquisição de conhecimento.2003. 163 f. Dissertação (Mestrado) -Curso de Engenharia de Produção, Escola de Engenharia de São Carlos da Universidade de São Paulo, São Carlos, 2003.

Paragon Decision Science - Arena.

Toledo, I. B., Jr. (2004). Lay-out: arranjo físico (8. ed.). Mogi das Cruzes: O\&M Itys Fides

NUNES, A.M. et al. Proposta de um o modelo de arranjo físico: estudo de caso numa panificadora em Campina Grande-PB. In: XXXII Encontro Nacional de Engenharia de Produção. Anais... Bento Gonçalves/RS, 2012.

ROSA, G. P.; CRACO, T.; REIS, Z. C.; NODARI, C. H. A reorganização do layout como estratégia de otimização da produção. GEPROS. Gestão da Produção, Operações e Sistemas, v. 9, n. 2, 2014.

MOTA, Kamila. Gestão por Processos. 6. ed. [S. I.: s. n.], 2014. 